\title{
The ventriloquist effect does not depend on the direction of deliberate visual attention
}

\author{
PAUL BERTELSON \\ Free University of Brussels, Brussels, Belgium \\ and Tilburg University, Tilburg, The Netherlands \\ JEAN VROOMEN \\ Tilburg University, Tilburg, The Netherlands \\ BÉATRICE DE GELDER \\ Tilburg University, Tilburg, The Netherlands \\ and Free University of Brussels, Brussels, Belgium \\ and \\ JON DRIVER \\ University College London, London, England
}

\begin{abstract}
It is well known that discrepancies in the location of synchronized auditory and visual events can lead to mislocalizations of the auditory source, so-called ventriloquism. In two experiments, we tested whether such cross-modal influences on auditory localization depend on deliberate visual attention to the biasing visual event. In Experiment 1, subjects pointed to the apparent source of sounds in the presence or absence of a synchronous peripheral flash. They also monitored for target visual events, either at the location of the peripheral flash or in a central location. Auditory localization was attracted toward the synchronous peripheral flash, but this was unaffected by where deliberate visual attention was directed in the monitoring task. In Experiment 2, bilateral flashes were presented in synchrony with each sound, to provide competing visual attractors. When these visual events were equally salient on the two sides, auditory localization was unaffected by which side subjects monitored for visual targets. When one flash was larger than the other, auditory localization was slightly but reliably attracted toward it, but again regardless of where visual monitoring was required. We conclude that ventriloquism largely reflects automatic sensory interactions, with little or no role for deliberate spatial attention.
\end{abstract}

When auditory and visual events, such as sound bursts and light flashes, occur close together in time but at somewhat disparate locations, source localization for one modality interacts with that for the other modality in characteristic ways (Bermant \& Welch, 1976; Bertelson, 1994, 1998; Radeau, 1994). These interactions are interesting because they may help us to understand the processes by which a coherent representation of extrapersonal

The present work was supported by the Ministry of Scientific Research of the Belgian French-Speaking Community (Concerted Research Actions 91/96-148 and 96/01-2037) and by the Belgian National Fund for Collective Fundamental Research (Contract 2.45.39.95). While working on this study, J.V. held a fellowship of the Royal Netherlands Academy of Arts and Sciences (KNAW). J.D. was supported by the Medical Research Council (U.K.). The assistance of the technical department of Tilburg University is gratefully acknowledged. Thanks are due to John H. Flowers, Ken W. Grant, Lester E. Krueger, and an anonymous reviewer for their constructive criticisms of earlier versions of the paper. Correspondence concerning this article should be addressed to P. Bertelson, Laboratoire de Psychologie Expérimentale, U.L.B., 50 Av. F. D. Roosevelt, B-1050 Bruxelles, Belgium (e-mail: pbrtlsn@ulb.ac.be).

-Accepted by previous editor, Myron L. Braunstein space is established and maintained. It has become customary to apply the collective term ventriloquism to all cases of auditory-visual spatial interaction, because the most familiar example from everyday life is the illusion created by performing ventriloquists, whereby the speech they produce without visible articulatory movements appears to come from a puppet they move in approximate synchrony with the speech sounds. A similar illusion is, of course, experienced by most movie audiences when they hear the spoken soundtrack as emanating from the actor's moving mouth on the screen. Several previous studies have considered whether such ventriloquism is due primarily to sensory factors or to more cognitive factors. In this article, we test, for the first time, whether ventriloquism depends on the direction of deliberate visual attention.

Ventriloquism has been formally demonstrated in many situations with spatial discordance between concurrent auditory and visual events and is revealed both by its online effects and by its aftereffects. The on-line effects include a cross-modal influence on unimodal localization, sometimes called crossmodal bias. ${ }^{1}$ This is shown when a subject is asked to localize the stimulation from just one of the two modalities (e.g., by pointing or by verbal report), 
while trying to ignore the stimulation in the other modality. Responses characteristically show partial displacement in the direction of the irrelevant competing stimulus from the other modality (Bermant \& Welch, 1976; Bertelson \& Radeau, 1981, 1987; Bertelson, Vroomen, Wiegeraad, \& de Gelder, 1994; Klemm, 1909; Radeau, 1992; Radeau \& Bertelson, 1976, 1987; Thomas, 1941; Warren, Welch, \& McCarthy, 1981). Most published demonstrations concern visual biasing of auditory localization, but there is evidence for a smaller auditory biasing of visual localization in some circumstances (e.g., Bertelson \& Radeau, 1981, Experiment 1; Radeau \& Bertelson, 1987).

A further on-line influence, perceptual fusion, can be observed when a subject mistakenly reports that the discrepant cross-modal stimuli originate from a single common location (Bertelson \& Radeau, 1981, Experiment 2; Choe, Welch, Gilford, \& Juola, 1975; Jack \& Thurlow, 1973; Witkin, Wapner, \& Leventhal, 1952). Finally, aftereffects can be observed when subjects perform localization for unimodal stimuli after a period of exposure to cross-modal discordance. In comparison with preexposure localization, postexposure localization is typically displaced toward the direction of the previous competing stimulus from the other modality, just as for on-line crossmodal bias (Canon, 1970; Radeau, 1992; Radeau \& Bertelson, 1974, 1976, 1977).

\section{Sensory Versus Cognitive Factors in Ventriloquism}

Many studies of ventriloquism have used fairly realistic situations, simulating real-life bimodal events, such as the voice of a person speaking and the concurrent sight of his or her face (e.g., Bertelson et al., 1994; Radeau \& Bertelson, 1977, Experiments 2-3; Warren et al., 1981; Witkin et al., 1952), the sight of whistling kettles (Jackson, 1953), or the sight of beating drums (Radeau \& Bertelson, 1977, Experiment 1). In such situations, the observed interactions might, to some degree, originate in the observer's prior knowledge of the simulated situations and, thus, may have a cognitive rather than a strictly sensory basis. However, ventriloquism is also found in simplified unfamiliar situations, such as those in which the inputs are reduced to meaningless sound bursts and flashes of light. Here, structural properties of the bimodal sensory input, such as the degree of temporal synchrony (Choe et al., 1975; Klemm, 1909; Radeau \& Bertelson, 1987; Thomas, 1941), spatial separation (Bertelson \& Radeau, 1981; Choe et al., 1975), or the relative saliency of the inputs (Radeau, 1985), constrain the cross-modal interactions.

Radeau and Bertelson (1977) compared the aftereffects of exposure to simplified versus realistic conflict situations. A voice reading a text was combined, in the realistic condition, with the sight of the speaker's face and, in the simplified condition, with light flashes synchronized to the amplitude peaks of the speech. Aftereffects of comparable sizes were produced in the two situations, suggesting that cognitive influences from a familiar context are not required for cross-modal adaptation to occur. ${ }^{2}$

On the other hand, Pick, Warren, and Hay (1969) reported a result that seems to demonstrate a role for purely cognitive factors in determining on-line cross-modal effects. Their observers, when pointing to the apparent source of a sound, shifted responses toward the discordant location in which a dummy loudspeaker was visible. This result was presumably a reflection of the knowledge that loudspeakers can deliver sounds. However, in a more recent experiment (Bertelson \& Radeau, 1987; Radeau, 1992), subjects exposed to a similar conceptual conflict situation showed no visual bias of auditory localization, whereas in a sensory conflict situation (with meaningless noise bursts and light flashes presented synchronously but at discrepant locations), they showed the usual visual influence. The fact that the cognitive influence reported by Pick et al. seems less reliable than that from synchronized sensory conflict suggests that the former influence may reflect postperceptual response biases, which are variable in nature.

Taken together, these previous results imply that the role of nonsensory factors in ventriloquism may be very limited at best. However, Welch and Warren (1980, 1984; see also Warren et al., 1981, and Welch, 1994) proposed that even the effects of stimulus factors, such as temporal synchrony and spatial separation, might themselves be mediated through judgmental cognitive processes, rather than through sensory processes. This notion received apparent support from an application of signal detection theory to findings concerning the effect of temporal synchrony on reports of audiovisual fusion (Choe et al., 1975). Choe et al: reported that synchrony apparently affected decision criteria, rather than perceptual sensitivity (i.e., $\beta$, not $d^{\prime}$ ), and concluded that ventriloquism was due to a cognitive response bias, rather than to a sensory change in perceived location.

However, close examination of Choe et al.'s (1975) method showed that the particular perceptual model they discounted was implausible from the outset. Bertelson and Radeau (1976) argued that a truly sensory effect of discrepant stimulation on unimodal localization could be reconciled with the signal detection data. On the other hand, they did not deny that strategic cognitive factors can influence responses in some audiovisual conflict situations (as for most phenomena based on observers' reports). Evidence for a genuinely sensory component to visual biasing of auditory localization has recently been reported (Bertelson \& Aschersleben, 1998), using a psychophysical staircase approach. This showed that the apparent location of sound bursts was attracted toward synchronous light flashes even when the subject showed no awareness of the spatial discrepancy, so that any judgmental factors were presumably minimized. 


\section{Possible Role of the Direction of Attention in Ventriloquism}

Our discussion so far has focused on the contrast between sensory accounts of ventriloquism as a genuine perceptual phenomenon and accounts that attribute it to more cognitive factors. There is, however, a third possibility, which has been generally ignored in previous discussions: an involvement of the direction of attention in the generation of ventriloquism. This possibility has become apparent in light of recent work on cross-modal links in spatial attention across hearing and vision (e.g., Driver \& Spence, 1994; Spence \& Driver, 1996), as we will discuss below.

Deliberate attention is not a strictly sensory factor, since it can be directed voluntarily under the observer's own cognitive control; but neither is it a strictly nonperceptual factor, since the attentional state of observers can affect their perception, not only their judgmental or decisional processes. Although the cognitive penetrabilility of perception still remains controversial with respect to top-down influences from knowledge (see, e.g., Krueger, 1989; Pylyshyn, 1999), the fact that deliberate attention can influence perception is no longer in dispute (see, e.g., Pashler, 1998, for an extensive review; also, Pylyshyn, 1999). Thus, considering the possible role of attention in ventriloquism should broaden the debate about the underlying mechanisms, beyond the simple sensory versus cognitive dichotomy that has been considered in the past.

In discussing how attention might influence ventriloquism, one must distinguish several different senses in which the term attention gets used. We may attend to one sensory modality versus another, regardless of location (Spence \& Driver, 1997b); or to one location versus another, regardless of modality (Spence \& Driver, 1996). Thus, modality selection can be distinguished from spatial selection. Moreover, for the case of spatial attention, we can use overt mechanisms of selection (i.e., shifting sensitive receptors toward a region of interest) or purely covert mechanisms (which enhance perception at particular locations even though no receptors shift; see Posner, 1980). Finally, our attention may be driven by the sudden onset of a salient stimulus, regardless of our intentions (exogenous attention capture); or may be directed deliberately, on the basis of expectations or task requirements (endogenous attention); see Yantis and Jonides (1990) for examples of this exgogenous/endogenous distinction. There is now considerable evidence suggesting qualitative differences between the operation of exogenous versus endogenous attention, both in cases of spatial selection (see Spence \& Driver, 1996, 1997a) and of modality selection (Spence \& Driver, 1997b). It is, therefore, important to keep these forms of attention distinct in any theoretical discussion.

Modality selection and its limits are clearly involved in classical demonstrations of ventriloquism. The fact that observers can be influenced by spatial location in one modality, even when asked to concentrate entirely on stim- ulation in another modality, indicates that modality selection is imperfect. Evidently, the irrelevant modality cannot be entirely filtered out, since it affects judgments of the target modality, despite instructions. Ventriloquism thus reveals a partial failure of endogenous modality selection. On the other hand, the failure is indeed only partial, since observers typically report different locations when instructed to localize either the visual or the auditory stimulation, and the sum of these biases is generally less than the actual spatial separation between the sources (Bertelson \& Radeau, 1981; Pick et al., 1969; Radeau, 1985, 1992; Warren et al., 1981). In an experiment in which both unimodal pointing and same-different origin judgments were recorded on every trial, Bertelson and Radeau (1981) found incomplete bias even on those trials on which perceptual fusion was reported. Thus, the reported location changes with modality instructions even for fused percepts. Mere instructions can apparently influence the weight given to data from the two modalities in the integration operation or, at least, their weight in the readout process that leads to the localization response. A related finding is that the aftereffects of exposure to audiovisual discrepancy, subsequently observed within either modality when tested alone, can be influenced by having the subject localize data from just one or the other modality during the bimodal exposure phase (Canon, 1970; Radeau, 1974).

Given this evidence for a role of deliberate (albeit imperfect) attention to one modality versus another in ventriloquism, one might ask whether deliberate spatial attention to one location rather than another plays any similar role. Some recent developments in cross-modal research on spatial attention may be taken to suggest such a possibility. Although in most of the attention literature, only a single modality at a time has been considered, a study by Driver and Spence (1994) demonstrated clear audiovisual links in endogenous spatial attention. They showed that unrelated auditory and visual tasks are combined less efficiently as a concurrent dual task when the auditory and visual stimuli have two spatially separate sources, rather than a common spatial position. This suggests a difficulty in directing endogenous visual and auditory attention to separate locations, rather than to a common location. In a related study, Spence and Driver (1996) reported that endogenous shifts of visual attention tend to be accompanied by corresponding shifts of auditory attention in the same direction, and vice versa.

These findings on cross-modal attention suggest the existence of an obligatory link between spatial attention in hearing and in vision. It thus seems possible that the biasing effects of vision on auditory localization, as found in many cases of ventriloquism, may, in part, reflect such attentional linkage between the two modalities. After all, real ventriloquists go to considerable lengths in their effort to get the audience to attend visually to the agitated puppet. Perhaps the observed influence of concurrent visual events on auditory localization depends on a shift in at- 
tention toward the synchronous visual events. As Spence and Driver (1996) found, such a shift in visual attention would tend to carry auditory attention along with it.

It should be noted that this suggested attentional account for ventriloquism actually involves a second premise beyond the demonstrated fact (Spence \& Driver, 1996, 1997a) that endogenous attention tends to shift together across the modalities. One would also have to assume that the locations registered within each modality depend, in part, on the current locus of deliberate attention within that modality (so that auditory localization would tend to shift along with attention toward a synchronous visual event). Several influential models of attention do, in fact, represent stimulus location by means of activity in an attentional master map, which represents the current focus of attention and, in so doing, also represents the perceived location that would be reported for the attended stimulus (see, e.g., Cave \& Wolfe, 1990; Treisman, 1988; Treisman $\&$ Gelade, 1980). Thus, given current findings on crossmodal attention and current models of how attention may relate to localization, the hypothesis that cross-modal links in spatial attention might underlie ventriloquism seems worthy of direct investigation.

In addition to its potential theoretical importance, the possible role of spatial attention in the generation of ventriloquism also has methodological implications for the design of experimental tasks. A common procedure in experiments on ventriloquism is to constrain the gaze of subjects via instructions to monitor a particular location for the possible occurrence of a prespecified visual target. For an appropriate interpretation of data obtained under such conditions, it is important to know whether such a monitoring requirement may, in itself, bias auditory localization toward the area of visual events, owing to an influence from the location at which attention is directed.

\section{The Present Study}

The experiments reported here test whether the effect of synchronous visual events on the apparent location of an auditory target may be influenced by the direction of deliberate attention toward, rather than away from, these synchronous visual events. The primary task was always to localize the apparent source of a sequence of sound bursts, which, in Experiment 1, could be accompanied by the synchronous flashing of an irrelevant visual square to one side, to serve as a potential visual attractor for inducing ventriloquism. In Experiment 2, synchronous flashes appeared on both sides, to provide competing visual attractors, and one could be more salient than the other. In both experiments, the secondary task was to monitor a small stream of visual events for occasional changes. The purpose of this was to manipulate the direction of deliberate visual attention. In Experiment 1, the visual monitoring task was performed either centrally or laterally at the location of the flashing attractor square. In Experiment 2, it was performed at the location of one or the other of the two lateral squares (i.e., always on one side, but now in the presence of bilateral visual attractors). The visual monitoring task involved both the deliberate focusing of visual attention on the location to be monitored and, also, overt gaze toward this location. If our manipulation of visual attention turned out to affect auditory localization, it would, of course, then be necessary to run further experiments, in order to separate the relative contributions of covert internal visual attention from those of overt gaze fixation. In fact, this necessity did not arise, because we found no influence of the direction of deliberate visual attention (and accompanying gaze) on auditory localization. All that finally mattered was the position and salience of the visual flashes presented synchronously with the sounds, implying that ventriloquism is not affected by deliberate attention but, rather, is determined by automatic sensory interactions.

\section{EXPERIMENT 1 Unilateral Visual Attractors}

In this experiment, each trial involved the presentation of a sequence of sound bursts at one of several locations (as determined by stereo presentation), plus the synchronous illumination on a computer screen of a square situated to the left or the right. The subjects pointed with an unseen (occluded) hand to the apparent origin of the sound. In addition, they had to monitor for rare small changes to a visual target (catch stimulus), which was located at the middle of the screen for one condition and at the middle of the peripheral flashing square for the other condition. If the direction of deliberate visual attention plays a role in ventriloquism, a larger shift of auditory localization toward the flashing square should be observed with lateral attention toward that square than with central attention.

\section{Method}

Subjects. Seventeen undergraduate students from Tilburg University were paid a small amount for participating in the $1 \frac{1}{2}-\mathrm{h}$ session of the experimental study.

Apparatus. The subject sat with his or her head on a chinrest at a 55-cm distance from a Commodore 1936 PC with a $20 \mathrm{~cm}$ high $X$ $27 \mathrm{~cm}$ wide monitor. All the visual input was displayed on this monitor. Two small loudspeakers were hidden behind an occluding screen on either side of the monitor. These allowed stereophonic presentation of the target sound bursts. The subject indicated the direction from which the sound appeared to emanate by moving the index finger of his or her preferred hand to the corresponding location along a bowed rod that was fixed flat to the table top in front of the screen. The main function of this rod was to serve as a distal boundary for the excursion of the pointing finger. A horizontal opaque panel hid the responding hand from the subject's sight. A graduated scale by the rod allowed the experimenter, who watched the finger through a video circuit, to record the pointing responses to the nearest degree of angle.

Procedure. On each trial, six 190-msec segments of $1000 \mathrm{~Hz}$ sine wave, each separated by 750 -msec silent intervals, were presented simultaneously over the two loudspeakers, at a comfortable listening level and with fade-ins and fade-outs, to avoid clicks. Three apparent locations of the sound were produced by setting the relative amplitude of the sine wave on the left and the right loud- 
speakers, respectively, at 100\%-100\% (middle), $110 \%-90 \%$ (left), and $90 \%-110 \%$ (right).

The center of the screen was occupied by a white circle, $10 \mathrm{~mm}$ in diameter, which came on at the beginning of each trial $(500 \mathrm{msec}$ before the onset of the first tone burst) and stayed on continuously until the offset of the last burst. On two thirds of the experimental trials, a $15 \times 15 \mathrm{~mm}$ white square, with its center at a distance of $82.5 \mathrm{~mm}$ $\left(8.5^{\circ}\right.$ of visual angle) to the left or the right of the screen center, flashed against a dark background in exact synchrony with the six sound bursts. The location of this attractor square was the same throughout the six cycles of each trial. Thus, the flashing square provided either a left or a right visual attractor that might influence auditory localization. On the remaining third of the experimental trials, no flashing square appeared. These no-square trials provided a baseline for estimating conflict-free auditory localization. The subject was instructed to perform the finger-pointing response for each trial only after the last of the six tones and to return the hand immediately thereafter to a near-medial resting position. The intertrial interval was $4 \mathrm{sec}$.

Separate blocks of trials were run under two different attention conditions. The critical difference between these conditions concerned the location of a small visual target, which had to be monitored for occasional slight changes in shape. The target always occurred in synchrony with the tones and in the same location throughout the six cycles of each trial. It was a small $(3 \times 3 \mathrm{~mm})$ black square, which on catch trials became a diamond (also $3 \times$ $3 \mathrm{~mm}$ ) on either the fifth or the sixth cycle of the trial. The instructions to the subject for these catch trials were to say "ja" (yes) and to abstain from pointing. Across different half-sessions, the subjects had to monitor visually at different locations. In the central attention condition, the visual target was situated, on all the trials, in the middle of the central circle. In the lateral attention condition, the visual target was presented in the center of the attractor square on the two thirds of the trials that had such a square and in the middle of the central circle on no-square trials. For the latter trials, the visual monitoring task was, thus, exactly the same as that for the corresponding trials in the central attention condition. Thus, direction of attention was only a dummy variable for no-square trials, denoting just the location on which attention was focused for those trials in the same half-session that did have an attractor square. The no-square trials served as baselines to assess the respective biasing effects of left versus right squares, but played no role in determining the effect of attention direction.

To check that monitoring for the catch stimuli effectively focused attention on the appropriate visual location in the experimental study, a separate control study was run on 4 subjects (who did not take part in the main experiment). They had to monitor for the same type of visual targets as that in the main experiment-that is, small black squares occasionally turning into black diamonds. The difference from the main experiment was that, in the control study, there were always two such visual streams, one on the central white circle and one on a lateral white square. The aim was to test whether visual monitoring at one location precluded visual monitoring at the other. If so, this would demonstrate the requirement for focused attention in the visual monitoring task.

In this control study, one block of trials was run under each of two conditions, which differed as regards the location (central or peripheral) that contained the majority $(75 \%)$ of the catch stimuli. The remaining $25 \%$ of the catch stimuli were presented in the other location, in a randomly intermingled sequence. The subjects in the control study were told of these probabilities and were requested to concentrate on the most frequent location for visual catch events, while attempting to detect all catch events at both locations. Their only task was to report catch events; although sounds were presented to make the stimulation as close as possible to that of the main experiment, no pointing to the location of these sounds was requested.
On each block of 96 trials in the control study, half (48) were catch trials, on which the critical change of a visual target from a square to a diamond occurred on either the fifth or the sixth cycle. The remaining half were no-catch trials. For the 36 catch stimuli occurring in the frequent location, the mean hit rate was $98 \%(96 \%$ for the central location and $100 \%$ for the lateral). Of the 12 catch stimuli in the infrequent location, none was ever detected by any subject. No false alarms occurred on no-catch trials.

These control results show that focusing attention at one of the two locations (central vs. lateral), in such a way as to be able to detect the target events in our visual monitoring task, made detection of similar events in the other location impossible. They therefore imply that the monitoring task focuses visual attention on a single location. It must be noted that this test of the extent to which attention must be focused in our monitoring task is a conservative one, since, in the control study, the subjects were, in effect, requested to monitor two locations, if possible, whereas in the main experimental study, they were requested to monitor only one visual location. Thus, the subjects in the experimental study should, if anything, have their deliberate visual attention even more focused.

Returning to the main experiment, lateral versus central attention was blocked by half-sessions, with order balanced across subjects. Each half-session included 90 experimental trials - that is, 10 under each combination of three sound locations (left, middle, right) crossed with three visual displays (square left, square right, no square), all intermingled with 12 additional catch trials and preceded by 3 additional warm-up trials.

Because lateral and central attention conditions were blocked, the subjects knew where to focus their deliberate visual attention. For the half-session in the central condition, they had to visually monitor the middle of the central circle throughout. For the halfsession in the lateral attention condition, they had to monitor the lateral attractor square when one was presented and the central circle when there was no attractor square. In any case, since the small visual target for monitoring occurred at the same location throughout the six cycles of each trial, its location on the first cycle would always inform the subject where to attend visually.

To summarize, on each trial in the experimental study, the subjects pointed to the perceived location of a sound, which could be presented at the right, at the left, or centrally. Large attractor squares could be presented on the left or the right, as potential inducers of ventriloquism that might influence auditory localization. At the same time, the subjects had to monitor small visual targets for occasional catch events, either centrally or at the location of the large attractor square. The control study had shown that this monitoring task requires attention to one visual location at the expense of the other. If ventriloquism depends on the direction of deliberate visual attention, the biasing effect of an attractor square on auditory localization should be more pronounced when subjects monitored for catch events at the peripheral location of the visual attractor, rather than centrally.

\section{Results}

Data from 5 subjects were discarded from the analysis because they missed more than 3 out of the 12 catch trials under one of the two monitoring conditions (in fact, under the lateral attention condition for all of them). For the remaining 12 subjects, the mean detection hit rate was $90 \%$ under the central attention condition and $93 \%$ under the lateral attention condition. Thus, there is little doubt that these subjects focused deliberate visual attention on the appropriate location with equal efficiency under both lateral and central conditions. 


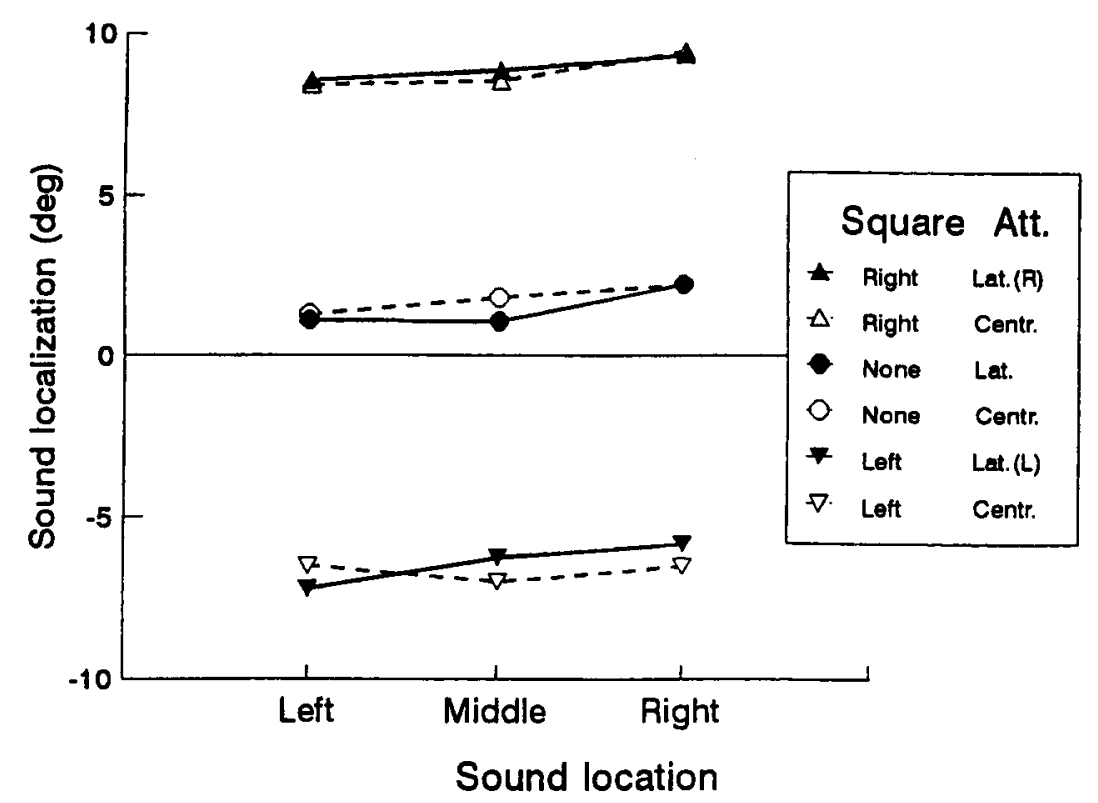

Figure 1. Experiment 1: Mean pointing response for sound localization as a function of sound location (along the abscissa), visual input (right flashing square, no square, or left flashing square; depicted by upright triangles at the top of the graph, circular symbols in the middle, or inverted triangles at the bottom of the graph, respectively), and visual attention condition (monitor at the central position, open symbols and dotted lines; monitor at the location of a lateral flashing square, filled symbols and solid lines). Sound localization, as measured by the pointing response, is shown along the $y$-axis, with zero representing the center of the scale. Negative values represent pointing toward the left, and positive values pointing to the right.

Figure 1 shows the mean pointing responses for all combinations of the three independent variables: sound location, visual input, and attention condition. The presence of a lateral square on the experimental trials resulted in strong shifts of pointing in its direction, as is shown by the large (vertical) differences between the three pairs of lines in Figure 1. By contrast, any influence of attention condition seemed very small and inconsistent (each solid line in Figure 1 is very close to the dotted line paired with it, which represents the other attention condition). As was discussed earlier, the results for the no-square trials (middle two lines) are not relevant to the question of the effect of attention direction, since, on those trials, attention was always, in fact, directed to the center. Any difference between the (dummy) attention conditions on these trials could only have arisen because of transfer from the task performed on the trials with an attractor square within the same half-session. The results for the trials with an attractor square are the relevant ones, and they show no effect of attention direction.

These results were statistically assessed by a three-way within-subjects analysis of variance (ANOVA). The main effects of sound location $[F(2,22)=7.3, p=.004]$ and of visual input $[F(2,22)=48.2, p<.001]$ were significant, but their interaction was not $[F(4,44)<1]$. There was no main effect of attention condition $(F<1)$, and its interactions with the other variables were all nonsignificant [attention $\times$ sound location: $F(2,22)<1$; attention $\times$ visual input: $F(2,22)<1$; attention $\times$ sound location $\times$ visual input: $F(4,44)=1.5$, n.s.]. The important outcome is the fact that the visual input $\times$ attention interaction did not even approach significance. Any influence of the direction of deliberate visual attention on ventriloquism would have shifted the pointing response in opposite directions for the trials with the square on the left and for those with the square on the right in the lateral attention conditions (as compared with central attention), which should have produced a significant interaction.

\section{Discussion}

In comparison with the control trials with no flashing square, pointing shifted on experimental trials in the direction of the lateral attractor square. This shift in auditory location occurred under both attention conditions and for all sound locations. Thus, the usual immediate biasing effect of synchronous but spatially discrepant visual events on auditory localization was obtained in the present situation; ventriloquism was produced.

The main focus of the experiment was any effect of requiring deliberate visual attention and the accompanying gaze to be directed either at the center of the display or, instead, at the flashing lateral attractor square that produced the cross-modal influence on auditory localization. No influence was observed from the direction of 
deliberate (endogenous) visual attention, suggesting that it plays little or no role in ventriloquism. In the General Discussion section, we consider whether the flashing attractors might have exerted their effect by capturing exogenous attention in an automatic manner, rather than by depending on endogenous attention. For now, we note merely that, if this were so, the results of Experiment 1 would imply that the deliberate direction of endogenous visual attention cannot modulate capture of exogenous attention by peripheral visual events. This would run contrary to several recent findings in the visual attention literature (see, e.g., Egeth \& Yantis, 1997; Yantis \& Jonides, 1990), which have demonstrated endogenous modulation of capture of exogenous attention.

The null effect of attention direction in Experiment 1 certainly cannot have been due to a failure to manipulate deliberate visual attention. The analyzed subjects effectively directed attention in the way requested by the instructions for the lateral versus central attention conditions, as is shown by their catch trial performance. Moreover, the results of the control study showed that such focusing of deliberate visual attention on one of the two locations produces a dramatic loss of detection performance in the other location.

However, there may be another respect in which Experiment 1 might have lacked sensitivity. One could argue that the attraction of auditory localization toward the single flashing square may have been so powerful that any more modest influence from deliberate attention may have been hidden. If so, it would be premature to conclude that the direction of deliberate attention never has any effect on ventriloquism, even though Experiment 1 clearly shows that it cannot be the sole determinant of the cross-modal effect. A more sensitive test could be provided by presenting flashing visual stimuli of comparable salience on both sides at the same time and then manipulating which side must be deliberately attended visually. In such a situation, we might now find that deliberate attention can influence which of two equally competitive visual attractors will come to dominate auditory localization. Experiment 2 tested this possibility.

\section{EXPERIMENT 2 Bilateral Visual Attractors}

This experiment was run to address the concern that the method used in Experiment 1 may have been insufficiently sensitive to reveal a role for deliberate spatial visual attention in ventriloquism, owing to dominance by powerful bottom-up factors. We now used a situation in which, in some of the conditions, two identical visual attractors were presented together, one on either side of the auditory input. In this case, no systematic bias of auditory localization would be expected to manifest itself from bottom-up visual stimulation. Any subtle influence of deliberate visual attention to one or the other side might then become more apparent. The experimental manipulation consisted of imposing deliberate visual attention to either the left or the right attractor, using the same monitoring task as that in Experiment 1.

If we found that the direction of deliberate attention had no effect even in such a situation, this would provide a much more convincing demonstration of the irrelevance of deliberate spatial attention to ventriloquism. On the other hand, one would also need to show that a situation with bilateral attractors is capable of producing some ventriloquism; otherwise, the sensitivity of our method might be questioned once again. Accordingly, we also introduced further bilateral conditions that had a stronger visual attractor on one side and a weaker one on the other side, in bottom-up stimulation terms. Radeau (1985) found that the biasing effect of a light flash on localization for a simultaneous sound burst is a function of the intensity of the light, with stronger effects from more intense lights. We expected that strength of attraction could similarly be manipulated in the present situation by varying the relative sizes of the visual squares flashed on each side. We predicted greater attraction of auditory localization toward the side of the larger square. When the squares were equivalent in size, any influence of deliberate attention on ventriloquism should be revealed by an analogous greater attraction to the side that was attended for the monitoring task. On the basis of Experiment 1, however, we now predicted no such effect from deliberate visual attention.

\section{Method}

The apparatus was the same as that in Experiment 1. The same sequence of six tones, with the same timing, was presented on each trial as before, and the primary task of the subject was again to point to its apparent source. Only two auditory locations, one left and the other right of the apparent middle, were used this time. The visual inputs were now always bilateral, comprising two squares flashing simultaneously with the tones on every trial, one on each side, with their centers again $82.5 \mathrm{~mm}$ from the center of the screen. Each square could be big (i.e., $15 \times 15 \mathrm{~mm})$ or small $(5 \times 5 \mathrm{~mm})$. By combining these two sizes, four possible visual arrangements were generated: small left/big right, or SB, plus BS, SS, and BB. Nosquare control trials, which had been run in Experiment 1, were omitted. Such trials are not necessary for testing the main hypothesis concerning any influence of deliberate attention on ventriloquism; in Experiment 1, their role had simply been to provide a baseline of pure auditory localization. To test any influence of deliberate visual attention on ventriloquism, all that is required is a comparison of localization responses for a given sound, when paired with concurrent attractor squares on both sides, with attention focused on the left versus the right attractor square.

The manipulation of deliberate visual attention involved the same monitoring task as that in Experiment 1. The same $3 \times 3 \mathrm{~mm}$ black square target was presented on each trial in the center of either the left or the right attractor square. On a catch trial, it became a 3 $\times 3 \mathrm{~mm}$ diamond on either the fifth or the sixth cycle. Unlike Experiment 1 , which had concerned central versus lateral attention condition, we now contrasted left and right visual attention. Moreover, whereas attention conditions had previously been blocked, attention to the left versus the right was now intermingled within each 


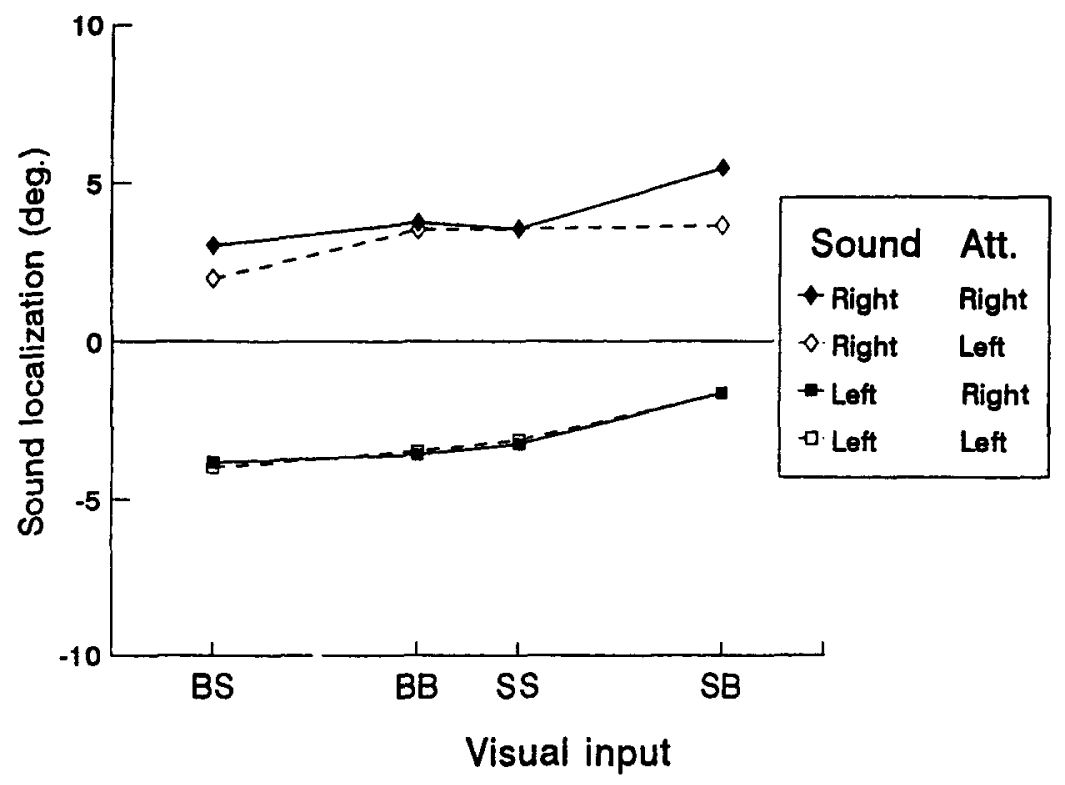

Figure 2. Experiment 2: Mean pointing response as a function of the different bilateral visual input (shown along the abscissa; BS stands for big square on the left, small square on the right, etc.), whether the sound was actually presented from the left (lower two lines in the graph, with squares) or from the right (upper two lines with diamonds), and whether visual monitoring was required on the right (filled symbols) or on the left (unfilled symbols). Sound localization, as measured by the pointing response, is shown along the $y$-axis, with zero representing the center of the scale. Negative values represent pointing toward the left, and positive values pointing to the right.

block. As the target for the monitoring task was again presented on the same side throughout the six cycles of each trial, the subject knew from the first cycle which side required deliberate visual attention.

Just as in Experiment 1, the subjects had to point to the apparent location of the sounds on all the experimental trials. On catch trials, where the small target square changed to a diamond, they had to withhold the pointing response and give a spoken response ("yes") to indicate their detection of the diamond.

Crossing the two possible sound locations (left/right), the four possible visual inputs (BS, SS, BB, and SB; i.e., the possible combinations of sizes for the single squares on each side, as was explained earlier), and the two visual attention requirements (monitoring at the left vs. the right square) yielded 16 different types of experimental trial. Each of these was presented twice, randomly intermingled with an additional four catch trials in each of five blocks of 36 trials each. Blocks were separated by a short pause. The session started with 16 practice trials.

Since the separation between the two possible locations for visual monitoring (now left vs. right) was twice as large in this experiment as that in Experiment 1 (where it had been lateral vs. central), we can assume that visually monitoring one location again precluded attending to the other, as was shown by the control study for Experiment 1.

Twenty-two undergraduate students from Tilburg University were paid to participate in the $1 / 2-h$ session. None had taken part in Experiment 1 .

\section{Results and Discussion}

Data from 3 subjects were discarded because they detected less than $75 \%$ of the catch stimuli. For the remaining 19 , mean hit rate on catch trials was $84.3 \%$.

The mean pointing responses were analyzed in a 2 (attention direction) $\times 2$ (sound location) $\times 4$ (visual input)
ANOVA, with all factors being within subjects. The main effects of sound location $[F(1,18)=23.9, p<.001]$ and of visual input $[F(3,54)=6.97, p<.001]$ were significant, but that of attention did not even approach significance $[F(1,18)<1]$. The sound location $\times$ attention interaction was also nonsignificant $[F(1,18)=1.52, \mathrm{n} . \mathrm{s}$.], as well as the other first-order interactions $(F<1)$, plus the sound location $X$ visual input $\times$ attention second-order interaction $(F<1)$.

The mean localization response for each combination of sound location, visual input, and direction of attention is shown in Figure 2 (note that the abscissa now denotes condition of visual stimulation, rather than that of auditory stimulation, unlike Figure 1). As would be expected, the main effect of sound location was due to rightward pointing for right sounds (upper lines, with diamonds, in Figure 2), as compared with leftward pointing for left sounds (lower lines with squares). Less trivially, the main effect of visual input appears to stem from the fact that, in comparison with the conditions with symmetrical visual input (i.e., SS and BB), the pointing responses in the asymmetrical conditions (i.e., SB and BS) are shifted in the direction of the bigger square (i.e., rightward and leftward, respectively). By $t$ test, the mean pointing responses in the two symmetrical conditions SS and BB were not significantly different $[t(18)<1]$. Their mean was used as a baseline for assessing the significance of any shifts toward the larger square in the asymmetrical conditions. Both shifts were significant by one-tailed $t$ tests [SB 
against baseline: $t(18)=3.56, p<.001$; BS against baseline: $t(18)=-1.83, p=.04]$. The use of one-tailed tests is justified by the fact that the direction of the shifts toward the larger square had been predicted.

As in Experiment 1, there was no consistent effect of the direction of deliberate attention in the monitoring task (in Figure 2, filled symbols with solid lines for attention to the right, open symbols with dotted lines for attention to the left). It is particularly noteworthy that there was absolutely no trend toward an effect of attention direction for conditions SS and BB of visual input, which should provide the most sensitive test for any influence of deliberate attention on ventriloquism, since these conditions have no bias favoring visual attraction of sound localization toward either side in purely bottom-up, stimulusbased terms. Thus, any top-down influence of deliberate attention to one side on ventriloquism would not have to compete with bottom-up factors in these conditions, unlike Experiment 1. Nevertheless, no effect of attention was observed even in this most sensitive situation.

The apparent trend toward a small influence of attention condition with BS and SB visual inputs for right sounds (apparent at either end of the upper two lines in Figure 2) is misleading. In fact, in the comparison of attend-left with attend-right for these conditions, the individual data went in the direction of the overall means for only 10 of the 19 subjects, and the overall difference was skewed by the data of just 1 subject (the same subject in both cases).

The most important finding of this experiment is that directing deliberate visual attention for the monitoring task to one side or the other had absolutely no reliable effect on sound localization. This was notably the case even for trials with symmetrical visual inputs (SS or BB), which should induce no bias toward one or the other side on a bottom-up basis, thus leaving ample room for any attentional effect to manifest itself. On the other hand, significant stimulus-driven visual biases on auditory localization were obtained in the two asymmetrical input conditions, always in the direction of the bigger square. This aspect of the results is important, because it shows that the experimental situation is sufficiently sensitive to let visual influences on sound localization manifest themselves. At a more general level, this finding confirms our assumption that the size of a visual stimulus can influence its capacity to bias auditory location, in the same way as previously shown for intensity (Radeau, 1985).

In passing, we note also that the biases of auditory localization toward the bigger square in conditions SB and BS were much smaller than those toward a single lateral square, as obtained in Experiment 1: about $1^{\circ}$, on average, in this study against about $7.5^{\circ}$ in Experiment 1 . It seems likely that, with bilateral visual inputs of different sizes, the observed shift in auditory localization represents the difference between the opposite attractions exerted by the two squares, rather than the total attractive power that the larger square would have exerted in isolation. This suggests that an averaging or competitive resolution of attractive power may take place between concurrent visual events, rather than ventriloquism being strictly a winnertakes-all phenomenon.

\section{GENERAL DISCUSSION}

As with many previous studies, in Experiment 1, it was found that the apparent location for a sequence of sound bursts is attracted in the direction of a visual event flashed synchronously in a discrepant location, the usual ventriloquist effect. The novel finding was that the size of this effect was uninfluenced by directing deliberate visual attention toward the location of the peripheral attractor flash (rather than to the center) in a concurrent visual monitoring task. Experiment 2 showed that directing attention deliberately to just one of two identical flashes, presented concurrently on either side, similarly exerted no influence on the apparent location of the sound source, even though such conditions of balanced bilateral stimulation should be maximally sensitive to any influence from the direction of deliberate attention. By contrast, increasing the size of one flash relative to the other one did produce an attraction of auditory localization toward the more salient of two bilateral visual stimuli. This pattern of results confirms bottom-up stimulus-based influences on ventriloquist phenomena, while suggesting that deliberate spatial attention plays little if any role.

Certainly, these results are inconsistent with any hypothesis that visual biasing of auditory localization, as found in ventriloquism, depends principally on where deliberate visual attention is directed. This seemed a possible account prior to our study, but the present results would seem to disconfirm it. Our findings fit more naturally with the alternative notion that ventriloquist phenomena reflect automatic interactions between sensory codes for location, arising at levels of representation that are preattentive, in the sense that they are uninfluenced by the current direction of deliberate attention in space.

The notion that ventriloquism may arise preattentively in this particular sense has received recent support from a demonstration that, in the traditional cocktail-party situation of trying to listen to just one of two competing spoken messages, ventriloquism can help listeners to focus on just the target message. Reisberg (1978) had originally shown that selective shadowing for one of two concurrent spoken messages can be improved by adding visual lip-read information for the relevant message. On its own, this result may show only that added visual stimulation can provide extra information about the content of the relevant message. However, Driver (1996) recently showed that seen lip movements can have a further specifically spatial influence on selective listening. He examined shadowing for one of two concurrent sets of spoken words, delivered by the same voice on a single mono loudspeaker, when visual lip-read information was now always presented for the relevant message. He found that shadowing was facilitated if the moving face of the person speaking was presented at a discrepant location, away from the mono sound source, rather than at the same position. 
This effect is probably due to a displacement of just the relevant sounds toward the location of the face, since the relevant sounds will be temporally correlated with the seen articulatory movements, whereas the distractor sounds will not. Ventriloquism in this situation should, therefore, displace the relevant sounds away from the distractor sounds, toward the moving face. Since this displacement can evidently aid selection of the target sounds from the distractor sounds, to produce more efficient shadowing, it must presumably arise prior to the operation of deliberate spatial attention. This conclusion accords with the implications of the present study, where ventriloquism was found to be unaffected by the direction of deliberate attention.

In laboratory situations such as those of the present experiment or those studied by Driver (1996), ventriloquism leads to mislocalization of auditory sources. However, in the real world, auditory sources typically will correspond in location to matching visual events, rather than being discrepant (e.g., speech sounds do, indeed, usually emanate from synchronously moving lips). Hence, the various cross-modal interactions that we have discussed can be viewed as reflecting mechanisms that should normally be adaptive, tending to favor veridical rather than illusory spatial perception. Given that auditory localization is generally rather inaccurate, relative to visual localization, it makes sense that visual location information should often be weighted more heavily than auditory location information, even for auditory judgments.

The aftereffects found after exposure to spatially discrepant auditory and visual stimulation, as was discussed earlier, suggest that auditory and visual spatial representations are continuously being recalibrated against each other. This presumably serves to optimize their correspondence with external reality, by updating their relation when consistent discrepancies arise (as has similarly been argued for the related prism-adaptation literature; see Held, 1965). From this perspective, it seems sensible that ventriloquist phenomena should, as we found, be unaffected by deliberate spatial attention. If auditory localization could be affected simply by wherever a person chose to attend visually, the apparent location of a fixed auditory source would change every time the person endogenously shifted his or her deliberate visual attention. Since the ultimate function of the cross-modal spatial interactions that underlie ventriloquist phenomena (including their aftereffects) is presumably to keep internal spatial representations in as close an accord with external reality as is possible, it would seem far more adaptive for these cross-modal spatial interactions to be driven passively by stimulus events in a bottom-up fashion, rather than being susceptible to wherever a person chooses to attend. Thus, our finding that deliberate spatial attention does not influence ventriloquism makes good functional sense.

We should delimit the restricted sense in which we propose that attention does not, and indeed should not, affect ventriloquist phenomena. First, our study has dealt only with spatial attention, not with attention to a modality. As was mentioned in the introduction, there are strong indications that audiovisual spatial interactions can be modulated to some extent by deliberate attention to one modality versus another, in contrast to what the present study has shown for deliberate spatial attention.

Second, our study has been concerned only with deliberate (or endogenous) spatial attention, directed in accordance with instructions to perform the monitoring task, and not with reflexive (exogenous) spatial attention, which may be captured by salient events in a stimulusdriven manner (see Egeth \& Yantis, 1997, Spence \& Driver, 1997a, and Yantis \& Jonides, 1990, for reviews of the endogenous/exogenous distinction for spatial attention). It remains an interesting question whether the automatic visual influence on auditory localization that was apparent in the present study (and in many previous experiments on the ventriloquist effect) might be mediated via exogenous spatial attention capture by the visual attractors.

The visual attractors in ventriloquist studies typically have abrupt onsets, as required to produce exogenous attention capture, according to Jonides and Yantis (1988). However, there are at least three difficulties for any interpretation of ventriloquism in terms of such exogenous attention capture alone. First, visual attractors that are asynchronous with sounds do not bias auditory localization (Bertelson \& Aschersleben, 1998; Klemm, 1909; Radeau \& Bertelson, 1987; Thomas, 1941), yet should induce exogenous capture equivalent to that produced by visual events that are synchronous with the sounds. Second, the present study has shown that the full ventriloquist effect occurs even with deliberate attention directed away from the visual attractor, a situation under which Yantis and Jonides (1990) have shown that exogenous capture no longer occurs. Thus, any suggestion that ventriloquism depends on capture of exogenous attention seems rather unlikely, given existing evidence. Finally, such an account would rely on an entirely conjectural influence from exogenous visual attention on auditory localization. Accordingly, for now, we prefer to stick closer to what was actually observed and to conclude merely that stimulus-driven interactions between the visual and the auditory events produce ventriloquism.

To summarize, the present experiments confirm earlier demonstrations that the visual biasing of auditory localization is strongly affected by stimulus factors, such as the relative location of auditory and visual events (Experiment 1) and the relative salience of concurrent visual events (Experiment 2), but they also show, for the first time, that the phenomenon is not affected by the direction of deliberate visual attention. We argue that this finding makes good functional sense. If deliberate changes in the direction of visual spatial attention were able to shift auditory localization for a fixed source in the corresponding direction, the senses might continuously recalibrate against each other, for apparent discrepancies that were, in fact, caused only by where the person had chosen to 
attend. Rather than there being such influences from endogenous spatial attention, we suggest that ventriloquist phenomena are largely driven, in an automatic fashion, by a coding of stimulus location that is preattentive, in the specific sense outlined above. This agrees with Driver's (1996) recent claim that ventriloquism may serve a useful segmentation function in noisy environments, regardless of where deliberate attention is currently directed. Finally, we acknowledge that ventriloquism may be subject to a different and nonspatial form of attentional controlnamely, a limited ability to weight the modalities differentially when making localization judgments. However, deliberate spatial visual attention to different positions seems to play little or no role in ventriloquism.

\section{REFERENCES}

Bermant, R. I., \& WELCH, R. B. (1976). The effect of degree of visualauditory stimulus separation and eye position upon the spatial interaction of vision and audition. Perceptual \& Motor Skill, 43, 487493.

BERTELSON, P. (1994). The cognitive architecture behind auditory-visual interaction in scene analysis and speech identification. Current $P$ sychology of Cognition, 13, 69-75.

BERTELSON, P. (1998). Starting from the ventriloquist: The perception of multimodal events. In M. Sabourin, F. I. M. Craik, \& M. Robert (Eds.), Advances in psychological science: Vol. 1. Biological and cognitive aspects (pp. 419-439). Hove, U.K.: Psychology Press.

Bertelson, P., \& Aschersleben, G. (1998). Automatic visual bias of perceived auditory location. Psychonomic Bulletin \& Review, 5, 482489.

Bertelson, P., \& Radeau, M. (1976). Ventriloquism, sensory interaction, and response bias: Remarks on the paper by Choe, Welch, Gilford, and Juola. Perception \& Psychophysics, 19, 531-535.

Bertelson, P., \& Radeau, M. (1981). Cross-modal bias and perceptual fusion with auditory-visual spatial discordance. Perception \& Psychophysics, 29, 578-584.

Bertelson, P., \& Radeau, M. (1987, September). Adaptation to auditory-visual conflict: Have top-down influences been overestimated here also? Paper presented at the 2nd Meeting of the European Society for Cognitive Psychology, Madrid.

Bertelson, P., Vroomen, J., Wiegeraad, G., \& de Gelder, B. (1994, September). Exploring the relation between McGurk interference and ventriloquism. In International Congress on Spoken Language Processing (pp. 559-562).

CANON, L. K. (1970). Intermodality inconsistency of input and directed attention as determinants of the nature of adaptation. Journal of Experimental Psychology, 84, 141-147.

CAVE, K. R., \& WolfE, J. M. (1990). Modelling the role of parallel processing in visual search. Cognitive Psychology, 22, 225-271.

Choe, C. S., Welch, R. B., Gilford, R. M., \& Juola, J. F. (1975). The "ventriloquist effect": Visual dominance or response bias? Perception \& Psychophysics, 18, 55-60.

DRIVER, J. (1996). Enhancement of selective listening by illusory mislocation of speech sounds due to lip-reading. Nature, 381, 66-68.

DRIVER, J., \& SPENCE, C. J. (1994). Cross-modal synergies in attention. In C. Umiltà \& M. Moscovitch (Eds.), Attention and performance XXV: Conscious and nonconscious information processing (pp. 311331). Cambridge, MA: MIT Press, Bradford Books.

Egeth, H. E., \& Yantis, S. (1997). Visual attention: Control, representation, and time course. Annual Review of Psychology, 48, 269297.

HELD, R. (1965). Plasticity in sensorimotor systems. Scientific American, 213, 84-94.
JACK, C. E., \& THURLOW, W. R. (1973). Effects of degree of visual association and angle of displacement on the "ventriloquism" effect. Perceptual \& Motor Skills, 38, 967-979.

JACKSON, C. V. (1953). Visual factors in auditory localization. Quarterly Journal of Experimental Psychology, 5, 52-65.

JONIDES, J., \& YANTIS, S. (1988). Uniqueness of abrupt visual onset in capturing attention. Perception \& Psychophysics, 43, 346-354.

KLEMM, O. (1909). Localisation von Sinneneindrücken bei disparate Nebenreizen [Localization of sense impressions with discordant additional stimulation]. Psychologische Studien, 5, 73-161.

KRUEGER, L. (1989). Cognitive impenetrability of perception. Behavioural \& Brain Sciences, 12, 769-770.

PASHLER, H. (1998). The psychology of attention. Cambridge, MA: MIT Press.

Pick, H. L., JR., Warren, D. H., \& HaY, J. C. (1969). Sensory conflict in judgments of spatial direction. Perception \& Psychophysics, 6, 203-205.

Posner, M. I. (1980). Orienting of attention. Quarterly Journal of Experimental Psychology, 32, 3-25.

PYLYSHYN, Z. W. (1999). Is vision continuous with cognition? The case for cognitive impenetrability of visual perception. Behavioural \& Brain Sciences, 22, 341-423.

RADEAU, M. (1974). Adaptation au déplacement prismatique sur la base d'une discordance entre la vision et l'audition [Adaptation to prismatic displacement on the basis of discordance between vision and audition]. L'Année Psychologique, 74, 23-34.

RADEAU, M. (1985). Signal intensity, task context, and auditory-visual interaction. Perception, 14, 571-577.

RaDEAU, M. (1992). Cognitive impenetrability in auditory-visual interaction. In J. Alegria, D. Holender, J. Morais, \& M. Radeau (Eds.), Analytic approaches to human cognition (pp. 41-55). Amsterdam: Elsevier.

Radeau, M. (1994). Auditory-visual interaction and modularity. Current Psychology of Cognition, 13, 3-51.

Radeau, M., \& BerTelson, P. (1974). The after-effects of ventriloquism. Quarterly Journal of Experimental Psychology, 26, 63-71.

Radeau, M., \& Bertelson, P. (1976). The effect of a textured visual field on modality dominance in a ventriloquism situation. Perception \& Psychophysics, 20, 227-235.

RadeAU, M., \& BerTelson, P. (1977). Adaptation to auditory-visual discordance and ventriloquism in semirealistic situations. Perception \& Psychophysics, 22, 137-146.

RAdEAU, M., \& BerTElson, P. (1987). Auditory-visual interaction and the timing of inputs: Thomas (1941) revisited. Psychological Research, 49, 17-22.

REISBERG, D. (1978). Looking where you listen: Visual cues and auditory attention. Acta Psychologica, 72, 331-341.

Spence, C., \& Driver, J. (1996). Audiovisual links in endogenous covert spatial attention. Journal of Experimental Psychology: Human Perception \& Performance, 22, 1005-1030.

SPENCE, C., \& Driver, J. (1997a). Audiovisual links in exogenous covert spatial orientation. Perception \& Psychophysics, 59, 1-22.

SPENCE, C., \& DrIVER, J. (1997b). On measuring selective attention to an expected sensory modality. Perception \& Psychophysics, 59, 389-403.

Thомas, G. J. (1941). Experimental study of the influence of vision on sound localisation. Journal of Experimental Psychology, 28, 167. 177.

Treisman, A. (1988). Features and objects: The fourteenth Bartlett memorial lecture. Quarterly Journal of Experimental Psychology, 40A, 201-238.

Treisman, A., \& Gelade, G. (1980). A feature-integration theory of attention. Cognitive Psychology, 12, 97-136.

Warren, D. H., Welch, R. B., \& McCarThy, T. J. (1981). The role of visual-auditory "compellingness" in the ventriloquism effect: Implications for transitivity among the spatial senses. Perception \& Psychophysics, 30, 557-564.

WELCH, R. B. (1994). The dissection of intersensory bias: Weighting for Radeau. Current Psychology of Cognition, 13, 117-123. 
WeLCH, R. B., \& WARREN, D. H. (1980). Immediate perceptual response to intersensory discrepancy. Psychological Bulletin, 88, 638-667.

WELCH, R. B., \& WARREN, D. H. (1984). Intersensory interactions. In K. R. Boff, L. Kaufman, \& J. P. Thomas (Eds.), Handbook of perception and human performance: Vol. I. Sensory processes and perception (chap. 25, pp. 1-36). New York: Wiley.

Witkin, H. A., WAPNer, S., \& Leventhal, T. (1952). Sound localization with conflicting visual and auditory cues. Journal of Experimental Psychology, 43, 58-67.

YANTIS, S., \& JONIDES, J. (1990). Abrupt visual onsets and selective attention: Voluntary versus automatic allocation. Journal of Experimental Psychology: Human Perception \& Performance, 16, 121-134.

\section{NOTES}

1. The term bias is used here, as it has been by other authors, in a purely descriptive way, to designate an observed influence. The use carries no assumption regarding the processing level at which the effect arises and, in particular, no implication as to whether response biases are involved, which we will discuss later.

2. In another experiment in the same study (Experiment 3), reports of perceptual fusion were enhanced by realism. On-line effects may be more susceptible to cognitive influences than are aftereffects, as we have previously suggested (Bertelson, 1998; Bertelson \& Aschersleben, 1998; Radeau \& Bertelson, 1977).

(Manuscript received August 21, 1997;

revision accepted for publication October 20,1998.) 